\title{
Infantile colic: maternal smoking as potential risk factor
}

Sijmen A Reijneveld, Emily Brugman, Remy A Hirasing

\begin{abstract}
The association of maternal smoking and type of feeding with colic was assessed in 3345 children aged 1-6 months (96\% response). The prevalence of colic was twofold higher among infants of smoking mothers, but less among breastfed infants. Maternal smoking as a potential risk factor for infantile crying needs further study.

(Arch Dis Child 2000;83:302-303)
\end{abstract}

Keywords: colic; preventive child health care; prevention; smoking; infancy

Infantile colic is a syndrome characterised by excessive, usually paroxysmal, crying in young, otherwise healthy, infants. ${ }^{1}$ The crying typically starts in the first weeks of life and ends at the age of about 6 months. Its aetiology remains unclear. It may have gastrointestinal and psychosocial causes, or it may simply be an extreme form of normal crying. ${ }^{1}$ Three studies have presented somewhat conflicting results on a potentially aetiological role of parental smoking. ${ }^{2-4}$ Matheson and Rivrud showed that breastfed infants of smoking mothers have colic more frequently. ${ }^{2}$ They explain this association by nicotine in maternal milk. ${ }^{5}$ Secondly, Said et al found an association with parental smoking independent from the type of feeding. ${ }^{3}$ Finally, Haggart and Giblin reported no association between colic and parental smoking. ${ }^{4}$ These conflicting results might be explained by small study sizes and the use of ambiguous definitions of colic. Our study examines the association of maternal smoking and type of feeding with the occurrence of colic in a large study, using a clear cut definition of colic.

\section{Methods}

Child health doctors and nurses interviewed the parents of a national sample of 3345 infants, stratified by age (table 1), on infant crying behaviour, maternal smoking, type of feeding, and background characteristics, in 1997 and 1998 (96\% response). Following Wessel, ${ }^{6}$ colic was defined as crying for more than three hours a day on more than three days of the preceding week. We assessed the association of maternal smoking and type of feeding with colic, using logistic regression.

\section{Results}

Colic occurred in $4.7 \%$ of all infants and was, with statistical significance, more frequent in younger infants and infants of smoking mothers (table 1). After adjustment for infant age, smoking was only associated with a higher prevalence of colic among completely formula fed children. Obstetric history and demographic and socioeconomic background did not explain these findings. Analyses that used another outcome, crying for more than three hours on only one day during the preceding week, yielded similar results (data not shown).

Analyses restricted to infants aged 1 month, the group with the highest overall prevalence of colic, yielded somewhat larger differences between groups. Odds ratios (OR) (95\% confidence intervals, CI) for smoking less than 15 cigarettes per day and for 15 and over, were 2.01 (1.18 to 3.42 ) and 2.85 (1.41 to 5.74 ), respectively, compared to infants of non-
Netherlands

Organisation of

Applied Scientific

Research (TNO),

Institute of Prevention

and Health, PO Box

2215, 2301 CE Leiden,

Netherlands

S A Reijneveld

E Brugman

R A Hirasing

Correspondence to:

Dr Reijneveld

email:

SA.Reijneveld@pg.tno.nl

Accepted 7 June 2000
Table 1 Prevalence of colic (\%) and odds ratios (OR) for colic regarding age of the infant, maternal smoking behaviour, and type of feeding

\begin{tabular}{|c|c|c|c|c|c|c|}
\hline Categories & No. ${ }^{\star}$ & $\%$ & Unadjusted $O R$ & p value & Adjusted OR† & $p$ value $\neq$ \\
\hline Age of infant $\S$ & & & & 0.000 & & \\
\hline 1 month & 1115 & 9.0 & 1.00 & & - & \\
\hline 3 months & 1085 & 3.7 & $0.39(0.27$ to 0.57$)$ & & & \\
\hline 6 months & 1120 & 1.3 & $0.14(0.08$ to 0.24$)$ & & & \\
\hline Maternal smoking & & & & 0.046 & & 0.015 \\
\hline Never & 2588 & 4.3 & 1.00 & & 1.00 & \\
\hline$<15$ cigarettes/day & 513 & 5.7 & $1.34(0.88$ to 2.04$)$ & & $1.50(0.97$ to 2.29$)$ & \\
\hline $15-50$ cigarettes/day & 182 & 8.2 & $2.00(1.14$ to 3.51$)$ & & $2.21(1.24$ to 3.92$)$ & \\
\hline Type of feeding & & & & 0.212 & & 0.053 \\
\hline Only formula & 2080 & 4.6 & 1.00 & & 1.00 & \\
\hline Mixed breast and formula & 365 & 3.3 & $0.71(0.39$ to 1.31$)$ & & $0.57(0.31$ to 1.06$)$ & \\
\hline Only breast & 871 & 5.5 & $1.22(0.85$ to 1.74$)$ & & $0.69(0.48$ to 1.01$)$ & \\
\hline Type of feeding by maternal smoking & & & & 0.146 & & 0.023 \\
\hline Never smokes & 2588 & 4.3 & 1.00 & & 1.00 & \\
\hline Smokes, only formula fed & 559 & 6.1 & 1.45 (0.97 to 2.15$)$ & & $1.81(1.21$ to 2.72$)$ & \\
\hline Smokes, (partially) breastfed & 164 & 6.1 & $1.45(0.74$ to 2.82$)$ & & $1.18(0.60$ to 2.32$)$ & \\
\hline
\end{tabular}

^Numbers do not always add up to 3345 due to missing values.

tOdds ratio adjusted for age of the infant.

$\neq \mathrm{p}$ value for inclusion of the characteristic in the logistic model, after adjustment for age of the infant

$§$ Age categories concerned the following intervals: 1 month $=3-5$ weeks; 3 months $=11-15$ weeks; 6 months $=22-30$ weeks. 
smoking mothers. However, compared with infants of non-smoking mothers, infants of smoking mothers that were breastfed had a less increased risk than completely formula fed infants: ORs are 1.61 (0.77 to 3.36) and 2.52 (1.50 to 4.25$)$, respectively.

\section{Conclusion}

Our study shows that maternal smoking and colic are associated but that breastfeeding weakens this association rather than reinforcing it.

It is unlikely that selective non-response has affected our findings because of the very high response rate. Some parents may not report their smoking, however, because of the warnings against it. This would give an underestimation of the strength of the association. However, another Dutch study that used mailed questionnaires yielded a similar prevalence of maternal smoking in infancy. ${ }^{\text {? }}$

Whether the association that we found can be explained by maternal smoking causing colic, needs additional study. Infantile crying may also have caused more maternal smoking, instead of the reverse. Furthermore, mothers who smoke during the infancy of their offspring may also have smoked during pregnancy, which also harms infant health, ${ }^{5}$ and might cause colic. Finally, smoking mothers may be more sensitive for crying, thus only reporting more crying, or may be less responsive so that their baby cries for longer.

Our findings regarding maternal smoking and breastfeeding confirm those of Said and coworkers, ${ }^{3}$ in a much larger sample. In contrast, Matheson and Rivrud only found an association with crying among breastfed infants, but their study concerned a mailed questionnaire survey four months postpartum (78\% response) and they defined colic less stringently. ${ }^{2}$ Finally, Haggart and Giblin reported an overall lack of association, but a reanalysis of their data yields an OR $(95 \% \mathrm{CI})$ for the association of parental smoking with colic of 1.66 (0.69 to 4.03$),{ }^{4}$ which fits with our findings.

If maternal smoking really causes colic, this may result in simple, though difficult to implement, ${ }^{57}$ advice to prevent colic: stop smoking. However, further research is needed to assess whether this association is indeed causal.

This study was financially supported by grants from the Dutch Ministry of Public Health and the Praeventiefonds (grant 28-2628-1).

1 Lucassen PLBJ, Assendelft WJJ, Gubbels JW, Van Eijck JTM, Van Geldrop WJ, Knuistingh Neven A. Effectiveness of treatments for infantile colic: systematic review. $B M \mathcal{F}$ 1998;317:1563-9.

2 Matheson I, Rivrud GN. The effect of smoking on lactation and infantile colic. $\mathcal{F A M}$ A 1989;261:42.

3 Said G, Patois E, Lellouch J. Infantile colic and parental smoking. BMF 1984;289:660.

4 Haggart M, Giblin MJ. Passive smoking and colic-like behaviour in babies. Health Visitor 1988;61:81-2

5 Haustein KO. Cigarette smoking, nicotine and pregnancy. Int F Clin Pharmacol 1999;37:417-27.

6 Wessel MA, Cobb JC, Jackson EB, Harris GS, Detwiller AC. Paroxysmal fussing in infancy, sometimes called "colic". Paediatics 1954;14:421-34.

7 Crone MR, Hirasing RA, Burgmeijer RJF. Prevalence of passive smoking in infancy in the Netherlands. Patient Educ Couns 2000;39:149-53. 